\title{
A Comment on the Paper "Systems with Variable Energy, Volume and Number of Particles: Evaluation of Partition Function and Thermodynamic Quantities" by I.M. Stanković, V.M. Marković, and Lj.Z. Kolar-Anić [1] ${ }^{1}$
}

\author{
Silvano Romano \\ Dipartimento di Fisica, Universitá di Pavia, Italy \\ e-mail: Silvano.Romano@pv.infn.it \\ Received April 7, 2013
}

DOI: $10.1134 / \mathrm{S} 0036024413120327$

The statistical-mechanical Ensemble for a fully open system (generalized Ensemble (gE) for short) discussed in the paper commented upon [1] was proposed by Guggenheim over seventy years ago [2], and discussed in detail by Prigogine and other authors as well (see, e.g., [3-6]); already in [2] it was pointed out that a generalized partition function for a single-component system only depending on intensive quantities $(T, P, \mu)$ has some "special" character: actually, by thermodynamics, $\mu$ is a function of $(T, P)$ via GibbsDuhem equation, i.e.,

$$
N d \mu=-S d T+V d P,
$$

where $N$ denotes the number of moles, $V$ and $S$ denote volume and entropy, respectively; the expression for $\mu(T, P)$ of an ideal monoatomic gas is a standard result in Statistical Thermodynamics [4, 6]. This aspect seems to have been overlooked in the paper commented upon [1].

The treatment of the named $\mathrm{gE}$ was related to, and partly intertwined with, another issue, i.e., a proper theoretical construction of the isothermal-isobaric Ensemble [2, 4, 5] (see also [6] and earlier ones quoted therein); early examples of actual calculations based on the gE are quoted in [6]; it was also discussed or

\footnotetext{
${ }^{1}$ The article is published in the original.
}

used for calculations in more recent years (see, e.g., [7-10]). Notice also that a detailed treatment of the named $\mathrm{gE}$ was carried out by Hill in [4], and later quoted in his book on Statistical Thermodynamics [7].

\section{REFERENCES}

1. I. M. Stanković, V. M. Marković, and Lj. Z. KolarAnić, Russ. J. Phys. Chem. A 85, 2257 (2011).

2. E. A. Guggenheim, J. Chem. Phys. 7, 103 (1939).

3. I. Prigogine, Physica 16, 133 (1950).

4. T. L. Hill, Statistical Mechanics, Principles, and Selected Applications (McGraw-Hill, New York, Toronto, London, 1956).

5. R. A. Sack, Mol. Phys. 2 , 8 (1959).

6. A. Münster, Statistical Thermodynamics (Springer, Berlin, Heidelberg, New York, Academic Press, New York, London, 1969), Vol. 1, Ch. 3, sects. 3.1 and 3.2.

7. T. L. Hill, An Introduction to Statistical Thermodynamics (Dover, New York, 1986), Ch. 1, sect. 1.7.

8. S. V. Shevkunov, A. A. Martsinovskii, and P. N. Vorontsov-Vel'yaminov, High Temp. 25, 176 (1988).

9. H. W. Graben and J. R. Ray, Mol. Phys. 80, 1183 (1993).

10. G. J. M. Koper and H. Reiss, J. Phys. Chem. 100, 422 (1996). 